\title{
Multifocal Pulmonary Abscesses due to Trueperella Pyogenes in Sheep
}

\author{
Harry Hariharan, Muhammad I. Bhaiyat, Keshaw P. Tiwari and Alfred Chikweto \\ Department of Pathobiology, School of Veterinary Medicine, St. George's University, \\ Grenada, West Indies
}

Correspondence should be addressed to: Harry Hariharan; hhariharan@sgu.edu

Received date: 25 March 2015; Accepted date: 11 May 2015; published date: 20 September 2016

Academic Editor: Nagy Gabor

Copyright (C) 2016. Harry Hariharan, Muhammad I. Bhaiyat, Keshaw P. Tiwari and Alfred Chikweto . Distributed under Creative Commons CC-BY 4.0

\begin{abstract}
An 8-year-old male sheep that was recumbent and in ill-health for over 7 days was euthanized. Necropsy revealed multifocal caseous abscesses in the cranial lobes of lungs, bilaterally. Microscopic findings included chronic suppurative bronchopneumonia with intralesional coccoid bacteria and multifocal granulomatous lymphadenitis of the tracheobronchial lymph node. Aerobic culture yielded heavy and pure growth of tiny, hemolytic colonies on blood agar, which were subsequently identified as Trueperella (Arcanobacterium) pyogenes, and confirmed using Analytical Profile Index (API) Coryne strips. Of 12 antimicrobial drugs tested against, including beta-lactam drugs: penicillin, ampicillin, and ceftiofur, the strain showed a susceptibility pattern typical of T. pyogenes, except for the resistance to trimethoprim-sulfamethoxazole. Published reports are sparse showing $T$. pyogenes as a cause of multiple pulmonary abscesses in sheep. This report provides evidence of this organism as a cause of extensive pathological changes in the lungs of an old sheep.
\end{abstract}

Keywords: Trueperella pyogenes, sheep, lung abscesses.

\section{Introduction}

Pneumonia is often diagnosed in adult sheep simply because the animal presents with an increased respiratory rate and breathing effort despite the fact that many infections affecting other organ systems can present with such signs including mastitis, metritis, clostridial diseases, and painful conditions such as lameness. There are three common causes of respiratory disease in sheep: respiratory disease caused by Mannheimia haemolytica, sheep pulmonary adenomatosis, and suppurative pneumonia/lung abscesses. Lung abscesses are not uncommon in adult sheep, but are difficult to diagnose on clinical examination alone. Sheep with lung abscesses generally show poor body condition and are often dull and depressed (NADIS, 2009).

Cite this Article as: Harry Hariharan, Muhammad I. Bhaiyat, Keshaw P. Tiwari and Alfred Chikweto (2016), "Multifocal Pulmonary Abscesses due to Trueperella Pyogenes in Sheep ", International Journal of Veterinary Medicine: Research \& Reports, Vol. 2016 (2016), Article ID 366083, 
Pyogenic infection in sheep is a serious health problem, and can even cause death of the affected animal (Al Dughaym, 2004). Pneumonia with pulmonary abscesses due to Pasteurella multocida, and Staphylococcus aureus, followed by Trueperella pyogenes, (formerly, Arcanobacterium pyogenes), Corynebacterium pseudotuberculosis, and Klebsiella pneumoniae have been reported in sheep (Anderson et al., 2002, Azizi et al., 2013).

Trueperella pyogenes is best known as an abscess-forming bacterium (Cebra and Cebra, 2002). This organism is one of the most common opportunistic pathogens in domestic ruminants. Brain abscesses in goats due to $T$. pyogenes has been recently reported (CAHFS, 2013). Although it is known that numerous abscesses including deeply seated abscesses can occur in ruminants (Jost and Billington, 2004), reports on multifocal lung abscesses in sheep are sparse. We report an uncommon case of multiple pulmonary abscesses due to Trueperella pyogenes in a sheep in Grenada, a tropical island nation.

\section{Case history and Pathological Diagnosis}

An eight year-old medium-built, male sheep weighing approximately $70 \mathrm{lbs}$ was in ill-health, and recumbent for 7 days. Because of the deteriorating condition, the owner elected euthanasia. This ram was one of a herd of seven that were routinely being dewormed with ivermectin oral drench. There was no history of previous disease in this ram but because it was unable to stand and its deteriorating condition, the owner elected euthanasia. Necropsy revealed moderate diffuse pulmonary edema and multifocal, caseous abscesses in the cranial lobes of lungs bilaterally, with pleural adhesions to the thoracic wall. The tracheobronchial lymph node was enlarged with the presence of multiple granulomas. There was moderate, bilateral osteoarthritis of the coxofemoral joint with roughened articular surfaces of the head of the femur and the acetabulum. No gross changes were detected in any other organs or tissues. Tissues were collected and preserved in 10\% formalin. Following routine processing and paraffin embedding, 4- $\mu \mathrm{m}$ sections were cut and stained with hematoxylin and eosin (H\&E). Histologically, there was chronic suppurative bronchopneumonia with infiltration by lymphocytes and macrophages, accompanied by cellular debris and bacterial colonies within the lumen of bronchioles and alveoli (Figure 1). Alveoli were filled with a lightly eosinophilic fluid and there was marked goblet hyperplasia in some bronchioles. There was diffuse lymphoid hyperplasia in the tracheobronchial lymph node with marked sinus histiocytosis. The granulomas (Figure 2) in the tracheobronchial lymph node were surrounded by a zone of necrosis and inflammation characterized by degenerate cells, cellular debris, dystrophic calcification and infiltration by lymphocytes, plasma cells, macrophages, multi-nucleated giant cells, and epithelioid cells. Bacterial colonies were present in the center, and a fibrous capsule around the periphery, of the granuloma. 


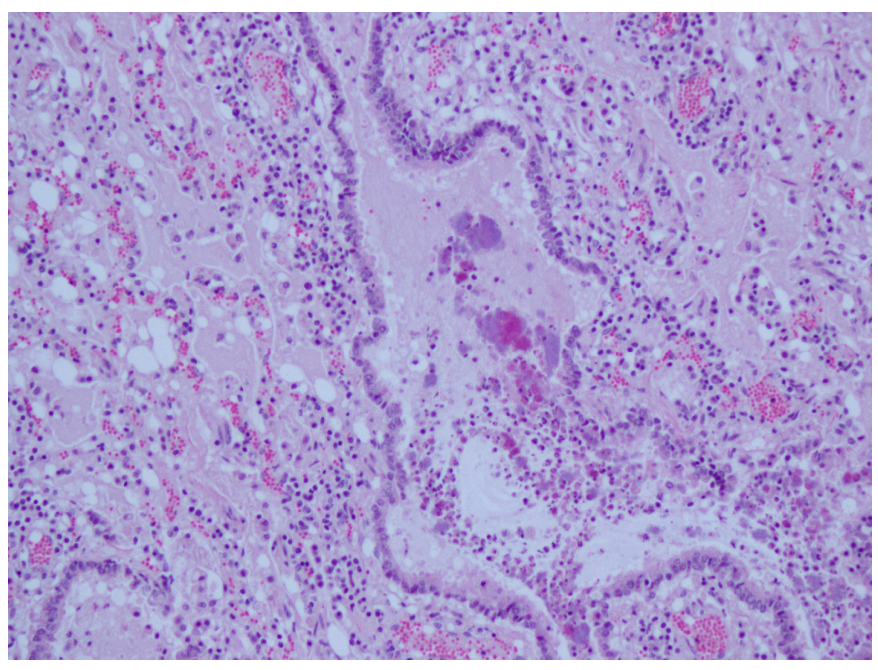

Figure 1: Lung, sheep; chronic bronchopneumonia

The bronchiolar epithelium is necrotic and the lumen contains neutrophils and macrophages admixed with cellular debris and bacterial colonies; alveoli spaces contain lightly pink edematous fluid admixed with lymphocytes and macrophages; chronic bronchopneumonia. (H\&E stain, X200)

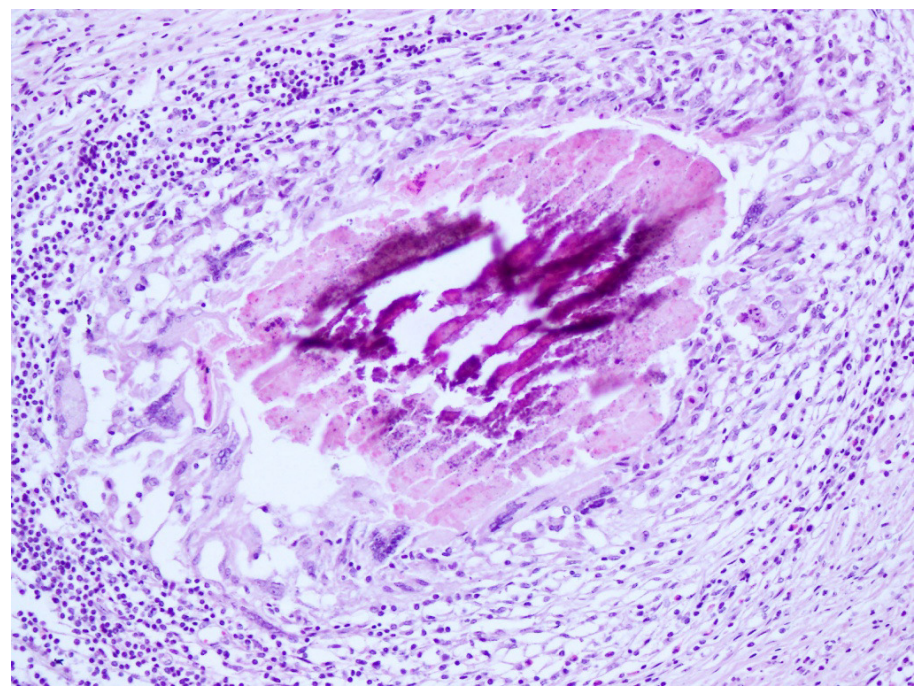

Figure 2: Tracheobronchial lymph node, sheep; granulomatous lymphadenitis Granuloma containing necrotic debris in the center and surrounded by macrophages, giant cells, and epithelioid cells in the periphery (H\&E stain, X200) 


\section{Bacteriological methods and Results}

Abscess contents from different areas of the lungs were aseptically collected and plated on blood agar and MacConkey media, and incubated aerobically at $37^{\circ} \mathrm{C}$. Culture plates were examined daily for 3 days. The growth was classified as heavy, moderate, and light. Three or less colonies were considered insignificant, and probably contaminants. The bacteria were identified based on colony morphology, Gram stain, classic biochemical tests, CAMP test (Quinn et al., 1994, Funke and Bernard, 2011), and by using a commercial bacterial identification system: "API Coryne" (bioMerieux Inc., Durham, NC, USA). Antimicrobial susceptibility tests were done against 12 drugs using the disk diffusion method as recommended by the Clinical and Laboratory Standards Institute (CLSI), and the zone sizes were interpreted as per CLSI guidelines (CLSI, 2008).

Heavy, pure growth of pinpoint colonies occurred from the samples after 24 hours of incubation, and after 48 hours the colonies were of $0.5 \mathrm{~mm}$ in diameter, and showed narrow zones of complete hemolysis around the colonies. Gram's smears showed Gram-positive, pleomorphic coccobacilli. Tests for catalase, oxidase, and urease gave negative results. The isolates gave positive CAMP reactions (Markey et al., 2013, Quinn et al., 1994). The isolates also gave positive fermentation reactions with glucose, lactose, maltose, and xylose, and negative reactions with sucrose and mannitol. Based on these results, the isolates were identified as Trueperella pyogenes. Inoculation of API Coryne strips resulted in identification as Arcanobacterium pyogenes (presently called Trueperella pyogenes). The isolates were susceptible to ampicillin, amoxicillin-clavulanic acid, ceftiofur, enrofloxacin, gentamicin, lincomycin, neomycin, penicillin, tetracycline, tilmicosin, and tulathromycin, and resistant to trimethoprim-sulfamethoxazole. Bacterial species other than $T$. pyogenes were not isolated. Considering the nature of lesions, we did not examine for viruses.

\section{Discussion}

Corynebacterium pyogenes (presently, Trueperella pyogenes) has been known to cause abscesses and mortalities in sheep in Iran (Tadayon et al., 1980). Foreyt and Jessup (1982) reported fatal pneumonia of bighorn sheep involving Pasteurella multocida and Corynebacterium pyogenes, following association with domestic sheep. Braun et al., (1995) described a two-yearold ram with severe chronic suppurative pleuropneumonia with abscess formation due to Actinomyces pyogenes (presently, Trueperella pyogenes) in Switzerland. This organism can cause pneumonia as well as sepsis in sheep (Cebra and Cebra, 2002, Songer and Post, 2005, Molecular Diagnostic Services, 2014). Heng and Holt (2007) reported from the United States a case of a large pulmonary abscess in a 3year-old Suffolk ram, which had decreased appetite, chronic weight loss and intermittent fever. The sheep did not respond to treatment with numerous antimicrobials, and had to be euthanized. Microscopic examination of the purulent fluid revealed numerous degenerative neutrophils and a mixed population of bacteria. Culture for bacteria was not performed. The case, however, showed the importance of pulmonary abscesses in sheep. Pulmonary abscessation is a common sequel to suppurative bronchopneumonia. Pulmonary abscesses may be present in many cases of pneumonia in animals and are not recognizable clinically. In the absence of pneumonia, abscessation is usually chronic, and clinical signs being produced are due to toxemia. When the spread is hematogenous, large numbers of small abscesses develop simultaneously. Most cases progress slowly and many affected animals have to be euthanized because of chronic ill-health (Radostits et al., 2007). In the present case, however, the inability of the ram to stand up could be attributable to the bilateral, coxofemoral osteoarthritis,

Overall, published information on lung lesions caused by Trueperella pyogenes, a common commensal organism of ruminants, is sparse. The present report 
brings attention to the fact that $T$. pyogenes can cause extensive abscessation in lungs. Suppurative bronchopneumonia is characterized by cranioventral consolidation of lungs which was observed in this case. It can be acute and fulminating, but is often chronic, depending on the etiologic agent, stressors affecting the host, and immune status. Most of the organisms causing suppurative bronchopneumonia are secondary pathogens requiring a preceding impairment of the pulmonary defense mechanisms to allow them to colonize the lung and establish an infection. The identification of isolates in the present study was confirmed by API bacterial identification strips. Funke et al., (1997) noted that Arcanobacterium pyogenes is one of the Arcanobacterium species correctly identified by the API Coryne system. Concerning antimicrobial drugs, our results showed the susceptibility of the organism isolated from abscesses to many drugs, including ceftiofur and tetracycline, which are used for respiratory infections in sheep (Navarre and Marley, 2006). Although the sheep in this study did not undergo antimicrobial therapy, it is known that systemic antimicrobial drugs can be used in sheep for deeply seated abscesses (Anderson et al., 2002). T. pyogenes is generally susceptible to beta-lactam drugs (Prescott, 2006a), and trimethoprimsulfamethoxazole (Prescott, 2006b). However, our strain was resistant to trimethoprim-sulfamethoxazole.

Antimicrobial susceptibility tests were done to determine if our strain showed a susceptibility pattern typical for $T$. pyogenes.

This report shows that Trueperella pyogenes, a commensal of sheep can cause extensive lesions in the lungs in these animals, and veterinarians should be aware of this bacterium, and the type of lesions it causes.

\section{Acknowledgements}

We thank Ms. Vanessa Matthew-Belmar, Erica Brathwaite, and Ms. Carla Richards for technical assistance.

\section{References}

1. Al Dughaym, A. M. (2004). "Isolation of Serratia, Arcanobacterium and Bukholderia species from visceral and cutaneous abscesses in four emaciated ewes," Veterinary Record, 155:425-426.

2. Anderson, D. E., Rings, D. M. \& Pugh, D. G. (2002). "Diseases of the integumentary system," In: Sheep and Goat Medicine. Editor: Pugh, D. G. W.B. Saunders Company, Philadelphia, PA. pp. 197-222.

3. Azizi, S., Korani, F. S. \& Oryan, A. (2013). "Pneumonia in slaughtered sheep in southwestern Iran: pathological characteristics and aerobic bacterial aetiology," Veterinaria Italiana, 49:109-118.

4. Braun, U., Fluckiger, M., Sicher., D. \& Theil, D. (1995). "Suppurative pleuropneumonia and a pulmonary abscess in a ram: ultrasonographic and radiographic findings," Schweizer Archiv fur Tierheilkunde, 137:272-278.

5. C. A. H. F. S. (2013). U. C. DAVIS: California Animal Health and Food Safety Laboratory System Connection: Small Ruminant Special Edition. http://cahfs.ucdavis.edu/localassets/pdfs/CAIHS_connection/CAHFS_con nection_small_ruminant_June_2013.pdf 6. Accessed 3 March, 2015.

7. Cebra, C. \& Cebra, M. (2002). “Diseases of the hematologic, immunologic, and lymphatic systems (multisystem diseases)," In: Sheep and Goat Medicine. Editor: Pugh, D. G. W.B. Saunders Company, Philadelphia, PA. pp. 359-391.

8. C. L. S.I. (2008). "Performance standards for antimicrobial disk and dilution susceptibility tests for bacteria isolated from animals; approved standard," Third Edition. Clinical and Laboratory Standards Institute; 2008.CLSI document M31-A3, Wayne, PA.

9. Foreyt, W. J. \& Jessup, D. A. (1982). "Fatal pneumonia of bighorn sheep following association with domestic 
sheep," Journal of Wildlife Diseases, 18:163168.

10.Funke, G. \& Bernard, K. (2011). "Coryneform Gram-positive rods," In: Manual of Clinical Microbiology (volume 1, Tenth Edition). Editors: Versalovic, J., Carroll, K.C., Funke, G., Jorgensen, J. H., Landry, M. L. \& Warnock, D.W. ASM Press, Washington, DC.pp. 413-442.

11.Funke, G., Renaud, F. N. R., Freney, J. \& Riegel, P. (1997). "Multicenter evaluation of the updated and extended API (RAPID) Coryne database 2.0," Journal of Clinical Microbiology, 35:3122-3126.

12.Heng, H. G. \& Holt, T. (2007). "What is your diagnosis? Pulmonary abscess," Journal of American Veterinary Medical Association, 230:993-994.

13.Jost, B. H. \& Billington, S. J. (2004). "Corynebacterium and Arcanobacterium," In: Pathogenesis of Bacterial Infections (Third Edition). Editors: Gyles, C. L., Prescott, J. F., Songer, J. G. \& Thoen, C. 0. Blackwell Publishing, Ames, Iowa. pp. 7786.

14.Markey, B. K., Leonard, F. C. \& Archambault, M. (2013). Clinical Veterinary Microbiology, (Second Edition), Mosby/Elsevier, New York, pp 151-154.

15.Molecular Diagnostic Services, 2014. Bacterial Diseases of Sheep. Http://www.mds-

usa.com/micro_bacteriology_sheep.html Accessed 3 March, 2015.

16.N. A. D. I. S. (2009). National Animal Disease Information Service. "Pneumonia in adult sheep," NADIS sheep health bulletin 17 December, 2009. http://www.fwl.co.uk/articles/17/12/200 9/119208/pneumonia-in-adult-sheepnadis-sheep-health-bulletin.htm

17.Navarre, C. B. \& Marley, S. (2006). "Antimicrobial drug use in sheep and goats," In: Antimicrobial Therapy in Veterinary Medicine, (Fourth Edition). Editors: Giguère, S., Prescott, J. F., Baggot, J. D., Walker, R. D. \& Dowling, P. M. Blackwell Publishing, Ames, Iowa, pp 519-528.

18.Prescott, J. F. (2006)a. "Beta-lactam antibiotics: penam penicillins," In: Antimicrobial Therapy in Veterinary Medicine, (Fourth Edition). Editors: Giguère, S., Prescott, J. F., Baggot, J. D., Walker, R.D. \& Dowling, P.M. Blackwell Publishing, Ames, Iowa, pp 121-137.

19.Prescott, J. F. (2006)b. "Sulfonamides, diaminopyrimidines, and their combinations," In: Antimicrobial Therapy in Veterinary Medicine, (Fourth Edition). Editors: Giguère, S., Prescott, J. F., Baggot, J. D., Walker, R. D. \& Dowling, P. M. Blackwell Publishing, Ames, Iowa, pp 249-262.

20.Quinn, P. J., Carter, M. E., Markey, B. \& Carter. G. R. (1994). Veterinary Clinical Microbiology, Mosby- Year Book Limited, London, England. p 150.

21.Radostits, O. M., Gay, C. C., Hinchcliff, K. W. \& Constable, P. D. (2007). Veterinary Medicine - A textbook of the diseases of cattle, horses, sheep, pigs and goats, (Tenth Edition), Elsevier, Philadelphia, PA. p 517.

22.Songer, J. G. \& Post, K. W. (2005). Veterinary Microbiology: Bacterial and fungal agents of animal disease. Elsevier Saunders, St. Louis, MO, USA. p. 59

23.Tadayon, R. A., Cheema, A. H. \& Muhammed, S. I. (1980). "Microorganisms associated with abscesses of sheep and goat in the south of Iran," American Journal of Veterinary Research, 41:798-802. 